\title{
Algunas cuestiones planteadas por el asesinato selectivo del general Soleimani
}

\author{
Mateo Fornari*
}

\section{RESUMEN}

El asesinato del general iraní, Qasem Soleimani, el 3 de enero de 2020 por un ataque aéreo estadounidense con dron, plantea algunas cuestiones de derecho internacional. Por lo que respecta al derecho de los conflictos armados, cabe preguntarse si esta acción es parte de un conflicto armado ya existente; si no existía un conflicto armado entre Estados Unidos e Irán antes del asesinato de Soleimani, cabe entonces preguntarse si esta misma acción, de por sí, ha provocado el comienzo de un conflicto armado entre los dos países. Establecido que EE. UU. e Irán no habrían actuado en el marco del jus in bello, cabe evaluar el asesinato de Soleimani en el marco del derecho del uso de la fuerza, es a decir del jus ad bellum. En este ámbito, la interpretación y aplicación del derecho de legítima defensa, invocado por los Estados Unidos suscita dudas.

Palabras clave: jus in bello, conflicto armado, jus ad bellum, legítima defensa; legítima defensa preventiva, legítima defensa precautoria.

\section{Some Questions Raised by the Targeted Killing of General Soleimani}

\section{Abstract}

The targeted killing of Iranian general, Qasem Soleimani, on 3 January 2020 by a drone strike by the United States, raises some international law issues. With regard to the law of armed conflict, it may be asked whether this action is part of an existing armed conflict; if there was no armed conflict between the United States and Iran prior to the assassination

\footnotetext{
* Profesor asociado de Derecho Internacional Público en el Departamento de Derecho de la Universidad de MilanoBicocca (2019) e investigador de Derecho Internacional Público en la misma universidad (2004-2019). Doctorado de Investigación de Derecho Internacional Público en la Universidad de Milano «La Statale» (2000) y licenciado en Derecho en la Universidad de Parma (1993). Titular del curso de Derecho de los Conflictos Armados (2010-2020). Correo electrónico: matteo.fornari@unimib.it
}

(iD) https://orcid.org/ 0000-0002-2028-9599 
of Soleimani, the question then arises as to whether this same action, in itself, has led to the beginning of an armed conflict between the two countries. Established that the United States and Iran would not be acting within the framework of the jus in bello, the assassination of Soleimani can be assessed in the context of the right to use force, that is, the jus ad bellum. In this context, the interpretation and application of the right of self-defence invoked by the United States raises doubts.

Keywords: jus in bello, armed conflict, jus ad bellum, self-defense, preventive self-defense, preemptive self-defense.

\section{Introducción}

La madrugada del 3 de enero de 2020, el general iraní Qasem Soleimani, jefe de la Fuerza $A l$-Quds, rama de la Guardia Revolucionaria iraní dedicada a operaciones exteriores, fue asesinado en Iraq por un ataque aéreo estadounidense con dron ordenado por el presidente Donald Trump — mientras viajaba en un convoy cerca del aeropuerto internacional de Bagdad. El ataque ocasionaba también la muerte de Abu Mahdi al Mohandes, vicepresidente de las Fuerzas de Movilización Popular (Hachd al Shaabi), milicias chiitas iraquíes pro-Irán, así como de otras siete personas. Para justificar este ataque, el mismo día el Departamento de Defensa de los Estados Unidos (EE. UU.) emitía un comunicado (U.S. Department of Defense, 2 de enero de 2020) según el cual:

U.S. military has taken decisive defensive action to protect U.S. personnel abroad by killing Qasem Soleimani, the head of the Islamic Revolutionary Guard CorpsQuds Force, a U.S.-designated Foreign Terrorist Organization. General Soleimani was actively developing plans to attack American diplomats and service members in Iraq and throughout the region. General Soleimani and his Quds Force were responsible for the deaths of hundreds of American and coalition service members and the wounding of thousands more. He had orchestrated attacks on coalition bases in Iraq over the last several months — including the attack on December $27^{\text {th }}$ - culminating in the death and wounding of additional American and Iraqi personnel. General Soleimani also approved the attacks on the U.S. Embassy in Baghdad that took place this week. This strike was aimed at deterring future Iranian attack plans. The United States will continue to take all necessary action to protect our people and our interests wherever they are around the world.

Siguiendo la posición de EE. UU., el asesinato de Soleimani se inscribiría, pues, en la lucha contra el terrorismo internacional iniciada en 2001 por la administración Bush: se trataba de un terrorista y además estaba preparando ataques inminentes contra intereses estadounidenses. El problema es que el «terrorista» era, además, jefe de las fuerzas armadas de otro país (la Fuerza $A l$-Quds es similar a una combinación entre la Agencia Central de Inteligencia y el Mando Conjunto de Operaciones 
Especiales de los EE. UU.) y número dos del régimen iraní: el equivalente sería que otro país hubiera matado al vicepresidente de los EE. UU.

El ataque estadounidense plantea algunas cuestiones, tanto desde la perspectiva del jus in bello como desde el punto de vista del jus ad bellum.

\section{El asesinato de Soleimani desde la perspectiva del derecho de los conflictos armados (jus in bello): acción como parte de un conflicto armado ya existente}

La muerte del general Soleimani no es el primer caso en que Estados Unidos ha asesinado (o intentado asesinar) a altos cargos político-militares de otro Estado. Por ejemplo, algo como un "asesinato selectivo» podría considerarse la muerte, durante la Segunda Guerra Mundial, del almirante Isoroku Yamamoto, jefe de la Flota Combinada de la Armada Imperial Japonesa, así como arquitecto y planificador del ataque contra la base naval estadounidense de Pearl Harbor en 1941. Tras haber interceptado por los servicios secretos de EE. UU. un mensaje en el que Yamamoto visitaría algunas bases japonesas en las Islas Salomón, el mismo presidente de los EE. UU., Franklin D. Roosevelt, aprobó la Operación Venganza, con la que aviones estadounidenses el 18 de abril de 1943, en los cielos de la isla de Bouganville, interceptaron y derribaron el avión en el que viajaba Yamamoto (Kelly, 1965, p. 102). Además, durante la guerra de Iraq de 2003 las fuerzas armadas estadounidenses estaban equipadas con barajas de cartas que representaban a los líderes políticos y militares del régimen iraquí para su identificación y posterior captura o eliminación (así, el jefe del régimen iraquí, Saddam Hussein, era el as de picas).

Pero en estos casos EE. UU. era parte de un conflicto armado y los jefes militares del Estado enemigo eran combatientes legítimos y, por ende, objetivo militar legítimo (así como, por supuesto, los jefes políticos cuando participen activamente y concretamente en la toma de decisiones estratégicas y en el mando de las fuerzas armadas durante un conflicto armado). Por ende, estas personas pueden ser objeto de ataques dondequiera que se encuentren, armados o desarmados, despiertos o dormidos, en primera línea o varias millas detrás de la línea del frente (Solis, 2007, p. 130; Dinstein, 2016, p. 42).

En este sentido, si hubiera una guerra entre Estados Unidos e Irán el general Soleimani iba ser un objetivo legítimo (aunque no estaba involucrado en ninguna acción armada en el momento de su muerte, y dejando aparte el hecho de que el ataque tuvo lugar en territorio iraquí). Por tanto, es necesario determinar si había una guerra (o más propiamente, un conflicto armado internacional, tal como lo define el Protocolo Adicional I de 1977 a los Convenios de Ginebra de 1949) entre EE. UU. e Irán en el momento del asesinato de Soleimani. 
De hecho, si se acoge la posición del Ministerio de la Defensa de EE. UU., el ataque contra el general iraní no parece una acción aislada sino una reacción armada consecuencia de una serie de acciones hostiles orquestadas por Soleimani, y conducidas por las Fuerzas iraquíes pro-Irán de Movilización Popular, contra bases estadounidenses en Iraq durante los meses anteriores. Estas acciones culminaron el 27 de diciembre de 2019 con el ataque por más de treinta cohetes contra la base aérea de Kirkuk (que albergaba al personal de la Operación Resolución Inherente) matando a un contratista civil estadounidense e hiriendo a cuatro miembros del servicio estadounidense y dos miembros del personal de las fuerzas de seguridad iraquíes (Garland, 28 de diciembre de 2019). Más aún, Soleimani habría aprobado también el ataque contra la embajada estadounidense en Bagdad la noche del 31 de diciembre por manifestantes iraquíes partidarios de las Brigadas del Partido de Dios (milicia fundada por al-Muhandis que, como se dijo, encontró la muerte junto a Soleimani). En verdad, el ataque a la embajada fue la consecuencia de los ataques aéreos del 29 de diciembre por parte de la Fuerza Aérea de EE. UU., que mataron al menos a 25 milicianos e hirieron a 55 más (U.S. Department of Defense, 29 de diciembre de 2019).

Con respecto a este escenario, dos cuestionen deben ser analizadas: en primer lugar, si esta serie de acciones violentas de ambas partes pueden considerarse prueba de la existencia de un conflicto armado entre EE. UU. e Irán; en segundo lugar, en caso de que la respuesta sea negativa, si el ataque contra Soleimani ha determinado la existencia de un conflicto armado, es decir, si el asesinato del general iraní por sí mismo ha dado lugar al comienzo de un conflicto armado y, por lo tanto, a la aplicación del derecho de los conflictos armados entre EE. UU. e Irán.

Por lo que respecta a la primera cuestión, hoy en día no cabe duda que no es necesaria la entrega de una declaración de guerra formal de un Estado a otro para que surja un conflicto armado entre dos países. De hecho, la práctica de los Estados demuestra que son más los conflictos armados que han surgido sin declaración de guerra que los que han nacido con este instrumento formal ${ }^{1}$. En este sentido, la ausencia de una

\footnotetext{
1 En 1907 los países participantes en la II Conferencia de la Haya (entre ellos EE. UU.) adoptaron el III Convenio de La Haya sobre el comienzo de las hostilidades que pedía una declaración de guerra formal para que empezara la aplicación del derecho de guerra (artículo 1). Este Convenio pronto pasó a ser obsoleto, demostrando la práctica incluso de los mismos Estados partes, cómo las guerras declaradas eran (y son) más que las declaradas formalmente. Por ejemplo, pueden mencionarse como guerras no declaradas la agresión de China por Japón en 1931, la agresión de Polonia por Alemania en 1939, el ataque aéreo de Japón contra EE. UU. en Pearl Harbor en 1941. Después de la Segunda Guerra Mundial, por ejemplo, puede mencionarse la guerra de las Falklands-Malvinas de 1982, entre Argentina y Reino Unido y la agresión de Kuwait por Iraq en 1990. Por supuesto, con la entrada en vigor de la Carta de las Naciones Unidas, una declaración de guerra se consideraría una violación de la prohibición del uso de la fuerza armada contra la integridad territorial o la independencia política de cualquier Estado (artículo 2, párrafo 4). Esto queda demostrado por el hecho de que usualmente los Estados, cuando hacen uso de la fuerza armada, invocan como causa da justificación la legítima defensa o la petición de ayuda del Gobierno del Estado atacado.
} 
declaración formal de guerra entre EE. UU. e Irán por supuesto no niega la existencia de una situación de conflicto armado entre los dos países.

Dicho esto, el hecho es que ni EE. UU. ni Irán nunca han declarado, por lo menos en estos últimos años, estar involucrados en un conflicto armado entre ellos. En los años ochenta del siglo pasado, la cuestión se planteó respecto del caso del derribo de un avión de línea iraní, el 3 de junio de 1988, por el crucero estadounidense USS Vincennes - presente en aguas del Golfo Pérsico- durante la guerra entre Iraq e Irán. Irán llevó la cuestión a la Corte Internacional de Justicia (Corte, o CIJ), alegando la violación del Convenio para la represión de actos ilícitos contra la seguridad de la aviación civil (Montreal, 1971) por parte de EE. UU.

Para sostener la inaplicabilidad de este Convenio, y por lo tanto su no violación, en la defensa presentada ante la Corte (ICJ, 2001, p. 203) EE. UU. argumentó que hubo conflicto armado con Irán:

Although it may be difficult to define in the abstract all of the circumstances that constitute an armed conflict, there is universal agreement that hostile operations carried out by military units of one country against the military units of another (such as was occurring between the military forces of the United States and Iran at the time that Iran Air Flight 655 was downed) constitute an armed conflict [...] the actions of the United States upon which Iran's claims in this case rest were taken by the military forces of the United States engaged in active hostilities with military forces of Iran. Under customary international law and relevant conventions, the actions of the parties in such a situation are governed by the laws of armed conflict.

No obstante, la argumentación estadounidense parece criticable. El crucero USS Vincennes cruzaba las aguas del Golfo para acompañar (y, si era necesario, defender) a los cargueros neutrales y, su posición era de estricta neutralidad con respecto al conflicto Iraq-Irán. Por ende, parece extrańo que ante a la Corte, EE. UU. argumentara estar involucrada en un conflicto armado contra Irán. Aún más si se considera que antes de que Irán recurriera a la Corte, EE. UU. nunca declaró ser parte de un conflicto armado con Irán. En otras palabras, el hecho de que EE. UU. sostuviera la existencia de un conflicto armado con Irán solo ante la CIJ parece más una «estrategia legal» que una convicción real. Y más, en octubre de 1987 y abril de 1988, EE. UU. atacó y destruyó unas plataformas petroleras de Irán. Contrariamente a lo que mantuvieron en el caso del derribo del avión iraní, esta vez EE. UU. no sostuvo la existencia de un conflicto armado con Irán; en cambio, destacó que estas acciones eran incidentes aislados y que EE. UU. no buscaba una confrontación con la República Islámica (ICJ, 2003) . Por otra parte, ni los demás países ni las Naciones Unidas han reconocido durante años que una situación de conflicto armado existiera entre EE. UU. e Irán, ni siquiera en el último periodo, antes del asesinato de Soleimani. Así pues, parece que hasta el ataque contra el general iraní faltaba un real 
animus bellandi de los dos países, por lo que se puede concluir en la inexistencia de un conflicto armado entre ellos.

Si no existía un conflicto armado entre EE. UU. e Irán antes del asesinato de Soleimani, cabe preguntarse entonces si esta misma acción, de por sí, provocó el comienzo de un conflicto armado y, por lo tanto, la aplicación del derecho de los conflictos armados entre los dos países. La cuestión que hay que establecer es si este uso de la fuerza armada tiene que alcanzar un cierto nivel de intensidad (¿o gravedad?) para determinar el inicio de la aplicación del jus en bello.

La legislación convencional pertinente no ofrece indicaciones al respecto. El artículo 2 común de los Convenios de 1949 indica simplemente que la normativa de Ginebra se aplica «en caso de guerra declarada o de cualquier otro conflicto armado» que surja entre dos Estados; así como el I Protocolo de 1977 solo especifica que su normativa "completa los Convenios de Ginebra» y se aplica "en las situaciones previstas en el artículo 2 común a dichos Convenios» (artículo 1, párrafo 3). A este respecto, también el Tribunal Penal Internacional para la ex-Yugoslavia en su decisión de 1995 sobre el caso Tadić, se limita a destacar como «an armed conflict exists whenever there is a resort to armed force between States» (ITFY, 1995, párrafo 70). Estos instrumentos no especifican si un ataque armado tiene que presentar un cierto umbral de intensidad (o gravedad) para que se pueda establecer que esta fuerza determina el comienzo de un conflicto armado. En otras palabras, no parece que la operatividad del jus in bello en caso de conflicto internacional sea sujeta a un determinado umbral de gravedad del conflicto.

En este orden de ideas se ha colocado también el Comité Internacional de la Cruz Roja. El comentario a los Convenios de Ginebra destaca cómo no es determinante la duración del conflicto ni el número de muertes: «It makes no difference how long the conflict lasts, or how much the slaughter takes place» (Pictet, 1952, p. 32). El mismo Comité, en su informe presentado en 2011 a su $31^{\circ}$ Conferencia Internacional (ICRC, 2011, p. 7), reiteró que en las décadas posteriores a la adopción de los Convenios de Ginebra, la duración o la intensidad de los combates no se consideraron elementos constitutivos de un conflicto armado internacional:

In the decades since the adoption of the Conventions, duration or intensity have generally not been considered to be constitutive elements for the existence of an IAC. This approach has recently been called into question by suggestions that hostilities must reach a certain level of intensity to qualify as an armed conflict.

La razón de la ausencia del elemento de la intensidad de los combates sería la de evitar controversias políticas y jurídicas sobre la determinación de la gravedad real de los combates en el caso concreto: 
It is submitted that, in addition to prevailing legal opinion which takes the contrary view, the absence of a requirement of threshold of intensity for the triggering of an IAC should be maintained because it helps avoid potential legal and political controversies about whether the threshold has been reached based on the specific facts of given situation.

Pero sin embargo debe tenerse en cuenta también que esta posición no es generalmente aceptada. La Asociación de Derecho Internacional (International Law Association, ILA), por ejemplo, subraya que el requisito de la intensidad de las hostilidades es un elemento necesario para la aparición de un conflicto internacional. Según su informe de 2010 sobre el significado de "conflicto armado» en derecho internacional, al menos dos características deben estar presentes en todos los conflictos armados (ya sean internacionales o internos): la existencia de un grupo armado organizado y el desarrollo de combates de cierta intensidad. Teniendo en cuenta la especial violencia que han caracterizado las múltiples guerras estalladas desde la Segunda Guerra Mundial, la ILA constata que «[t]he violence must be organized and intense - even between sovereign states - before the otherwise prevailing peacetime rules are suspended» (ILA, 2010, p. 2).

En este orden de ideas (y debido a la falta de indicaciones sobre el nivel necesario de violencia que determina el estallido de un conflicto armado), por lo que respecta al caso aquí considerado, ¿el ataque a Soleimani presenta un nivel de intensidad que permita afirmar que esta acción determinó el comienzo de un conflicto armado entre EE. UU. e Irán? La respuesta no parece positiva. De hecho, el modo en que se realiza un ataque así llamado "selectivo" en realidad parece demostrar la voluntad de quien lo realiza de minimizar los daños y las consecuencias materiales de este uso de la fuerza armada. Pero, más que el nivel de intensidad (no) alcanzado, se podría considerar como elemento que determina el comienzo de un conflicto entre dos países la gravedad de la acción armada, es decir, el asesinato de un objetivo de alto valor. En otras palabras, cuanto mayor sea el nivel político-militar de la persona asesinada, mayor sería la probabilidad de que surja un conflicto armado entre dos países. En este sentido, más que tener en cuenta la intensidad del ataque, se podría argumentar que el asesinato del número dos del régimen iraní dio lugar a un conflicto armado entre EE. UU. e Irán.

No obstante, tampoco esta segunda hipótesis parece aceptable en el caso que nos ocupa, por el hecho de que ninguno de los dos países ha declarado expresamente que se siente implicado en un conflicto armado tras el asesinato de Soleimani. El mismo Presidente Trump declaró, en una conferencia de prensa el 4 de enero, que EE. UU. «did not take action to start a war» contra Irán (Loveluck, Taylor y Brice-Saddler, 4 de enero de 2020); del mismo modo que el Gobierno iraní nunca ha declarado estar 
involucrado en un conflicto armado contra Estados Unidos. Como lo ha destacado Agnès Callamard, relator especial sobre las ejecuciones extrajudiciales, sumarias o arbitrarias del Consejo de Derechos Humanos de las Naciones Unidas, «no State, expert commentator or expert body, such as the International Committee of the Red Cross, had identified the escalation of the conflict between the U.S. and Iran as amounting to an international armed conflict» (Callamard, 8 de enero de 2020).

Por el contrario, el embajador iraní en una carta dirigida al Consejo de Seguridad el 8 de enero para justificar bajo la legítima defensa el lanzamiento de algunos misiles por Irán contra las bases estadounidenses en Iraq, declaró que su país «no busca la escalada de las tensiones ni la guerra» (Documento NU S/2020/19, 8 de enero de 2020). Por lo tanto, también en esta hipótesis parece el animus bellandi desempeñar un papel determinante para determinar si existe o no un conflicto armado entre los dos países.

Además, la no existencia de un conflicto armado entre EE. UU. e Irán se podría también demostrar por otras consideraciones. Por un lado, por lo que declaró un funcionario del Departamento de Estado estadounidense en una conferencia de prensa, el 4 de enero (Gilsinan, 4 de enero de 2020; cursiva añadida), sobre el hecho de que Soleimani fue asesinado porque no había otra forma para poner fin a sus actividades hostiles:

Do you have some legal means to, like, have this guy arrested by the Belgian authorities or something? Check that box, because there's no way anybody was going to stop Qassem Soleimani in the places he was running around - Damascus, Beirut. And so you take lethal action against him.

El funcionario no especificó a qué tipo de detención se sometería Soleimani; pero por el tono de esta declaración no parece que se pueda deducir que el general iraní hubiera sido considerado prisionero de guerra; por tanto, esta declaración parece una demostración de que EE. UU. no se consideraba parte en un conflicto con Irán.

Por otro lado, cabe señalar que el día que mataron a Soleimani, este se encontraba en Bagdad porque tenía que encontrar al primer ministro de Iraq, Adil Abdul-Mahdi, para entregarle un mensaje de Irán en respuesta a un mensaje anterior que Iraq transmitió a Teherán por parte de Arabia Saudita. Según lo declarado por el propio AbdulMahdi, Iraq estaba actuando como intermediario entre Irán y Arabia Saudita para facilitar la reanudación de los contactos entre los dos países (Villalaz, 21 de enero de 2020), así que el día de su asesinato Soleimani estaba en una misión diplomática para rebajar tensiones entre ellos, y no actuando para planear acciones ofensivas contra EE. UU. 
Lo dicho anteriormente parece llevarnos a concluir que el asesinato de Soleimani no dio lugar a un conflicto armado entre EE. UU. e Irán, y tampoco se llevó a cabo dentro de un conflicto ya en curso, por lo menos en el sentido del I Protocolo de 1977. Pero, a partir del «caso Soleimani» se podrían presentar algunas hipótesis a más largo plazo. Suponiendo que existe un conflicto de baja intensidad entre dos Estados, para que se aplique el derecho humanitario parece insuficiente el recurso a la fuerza armada, en tanto sería también y sobre todo necesario una manifiesta y expresa declaración formal de las autoridades gubernamentales que expresen la convicción de que se consideran parte de un conflicto armado. Esto no quiere decir que sea necesaria una declaración formal de guerra, pero al menos unas declaraciones o toma de posición oficial de personalidades político-militares de los Estados implicados en el conflicto. Por supuesto, en el caso de un conflicto armado de alta intensidad (es decir, una verdadera guerra), la existencia de dicho conflicto no estaría vinculada a declaraciones oficiales de los Jefes de Estado.

Por último, debe tenerse en cuenta que el ataque a Soleimani se llevó a cabo en territorio iraquí sin aprobación de Iraq, y además murió también Abu Mahdi al Mohandes, vicepresidente de las Fuerzas de Movilización Popular (Hachd al Shaabi), milicias chiitas iraquíes pro-Irán. En 2016, el Parlamento iraquí aprobó una ley por la que se incorporó este grupo armado a las fuerzas armadas iraquíes (Smith, Singer-Emery, 2018, p. 167 ss.; Smith, 5 de enero de 2020); por lo tanto, EE. UU., asesinando a Mohandis, liquidó a un agente del Estado iraquí. Entonces, cabría preguntarse si esta acción dio lugar también a un conflicto armado entre EE. UU. e Iraq. Es verdad que el mismo día del ataque, el primer ministro de Iraq calificó esta acción como "aggression» $\mathrm{y}$ "violation of the sovereignty of Iraq" (Al-Mahdi, 3 de enero de 2020); pero los días siguientes el propio primer ministro, en una reunión con el embajador de EE. UU., se aseguró de aclarar que «Iraq is making all possible efforts to prevent the slide of an open war» (Al-Mahdi, 6 de enero de 2020). También en este caso, los dos países nunca hicieron declaraciones o toma de posición oficial en que afirmaran la existencia de un conflicto armado entre ellos.

\section{El asesinato de Soleimani desde la perspectiva del jus ad bellum}

Establecido que EE. UU. e Irán no actuaron en el marco del jus in bello, cabe evaluar el asesinato de Soleimani en el marco del derecho del uso de la fuerza, es decir del jus ad bellum.

Como es bien sabido, la regla básica del jus ad bellum es la prohibición del uso de la fuerza armada, una norma imperativa codificada en el artículo 2, párrafo 4, 
de la Carta de las Naciones Unidas ${ }^{2}$. La Corte Internacional de Justicia, en el caso Nicaragua-EE. UU., destacó «the validity as customary international law of the principle of the prohibition of the use of force expressed in Article 2, paragraph 4», y que «the law of the Charter concerning the prohibition of the use of force in itself constitutes a conspicuous example of a rule in international law having the character of jus cogens» (ICJ, 1986, párrafo 190). La única excepción posible a la prohibición del uso de la fuerza es la legítima defensa ${ }^{3}$, «derecho inmanente» — es decir un derecho de valor consuetudinario - como subraya el artículo 51 de la Carta:

Ninguna disposición de esta Carta menoscabará el derecho inmanente de legítima defensa, individual o colectiva, en caso de ataque armado contra un Miembro de las Naciones Unidas, hasta tanto que el Consejo de Seguridad haya tomado las medidas necesarias para mantener la paz y la seguridad internacionales. Las medidas tomadas por los Miembros en ejercicio del derecho de legítima defensa serán comunicadas inmediatamente al Consejo de Seguridad [...]

Además, como lo ha destacado la CIJ, el recurso a la fuerza armada en legítima defensa debe ejercerse dentro de ciertos límites de valor consuetudinario, es decir que tiene que respetar los requisitos de necesidad, proporcionalidad e inmediatez (ICJ, 1986, párrafo 176; ICJ, 2003, párrafo 76).

Un tema muy delicado del artículo 51 se refiere a la dimensión temporal del «ataque armado", es decir la cuestión de establecer cuándo surge el derecho del Estado expuesto a ataque a reaccionar en legítima defensa. El dato literal del artículo 51 indicaría una interpretación restrictiva, ya que parece vincular el ejercicio del derecho de legítima defensa a la actualidad del ataque armado, o sea a un ataque actualmente en curso ${ }^{4}$. En ese orden de ideas se colocó también la CIJ, en dos casos que involucraron a EE. UU.: en el caso Oil Platforms la Corte destacó que EE. UU. tenía que demostrar que los ataques llevados a cabo por Irán «were of such nature as to be qualified as 'armed attacks' within the meaning of that expression in Article 51 [...] and as understood in customary law on the use of force»(ICJ, 2003, párrafo 51); y en el caso Military Activities in Nicaragua, subrayó que «[i]n the case of individual self-defence, the exercise of this right is subject to the State concerned having been the victim of an armed attack» (ICJ, 1986, párrafo 195).

\footnotetext{
2 Artículo 2, párrafo 4 de la Cartas de las Naciones Unidas: «Los Miembros de la Organización, en sus relaciones internacionales, se abstendrán de recurrir a la amenaza o al uso de la fuerza contra la integridad territorial o la independencia política de cualquier Estado, o en cualquier otra forma incompatible con los Propósitos de las Naciones Unidas».

3 El uso de la fuerza puede ser recomendado por el Consejo de Seguridad a los Estados cuando determine una amenaza a la paz, un quebrantamiento de la paz o un acto de agresión, sobre la base de una interpretación «extensiva» del Capítulo VII, y en particular del artículo 42 de la Carta.

4 Véase también el texto inglés («if an armed attack occurs against a Member of the United Nations») y el texto francés («dan le cas où un Membre des Nations Unies est l'objet d'une aggression armée»).
} 
A pesar de las indicaciones de la Corte, la práctica de algunos países -EE. UU. en primer lugar - pone también en evidencia una interpretación extensiva del instituto de la legítima defensa, admitiendo la legítima defensa "preventiva», en virtud de la cual un Estado expuesto al peligro de un ataque armado seguro e inminente, pero aún no iniciado, podría empezar una acción defensiva para evitar dicho ataque. Lo que caracteriza el concepto de legítima defensa preventiva es, por tanto, la dimensión temporal y la certeza de la «inminencia».

Sin embargo, en la última década EE. UU. ha formulado - y aplicado — una versión de la legítima defensa aún más permisiva, es decir la legítima defensa "precautoria» (pre-emptive self-defence). Esta versión de legítima defensa aparece «gracias» a la Administración Bush Jr., en un documento de 2002 — The National Security Strategy of the United States of America — que "ponía las bases» para luchar contra el fenómeno del terrorismo internacional (y que, en verdad, servirá a EE. UU. para «justificar» la agresión de Iraq en 2003). La parte que interesa aquí (The National Security Strategy of the United States of America, 2002, p. 15; cursiva ańadida) - y que, evidentemente, sigue interesando a la Administración Trump - afirma que:

The United States has long maintained the option of preemptive actions to counter a sufficient threat to our national security. The greater the threat, the greater is the risk of inaction-and the more compelling the case for taking anticipatory action to defend ourselves, even if uncertainty remains as to the time and place of the enemy's attack. To forestall or prevent such hostile acts by our adversaries, the United States will, if necessary, act preemptively.

Por lo tanto, EE. UU. justifica una respuesta armada para evitar la mera amenaza o la simple conjetura de futuros o potenciales ataques armados: con la pre-emptive self-defence no existe la certeza y la inminencia del ataque (que caracteriza la legítima defensa «preventiva»). Así, con la legítima defensa "preventiva» o con la "precautoria» un país emplea la fuerza armada antes de ser atacado, pero con la «preventiva» tiene la certeza de que un ataque inminente contra él se está produciendo, mientras que con la "precautoria» falta totalmente esta certeza. En otras palabras, con esta figura de legítima defensa falta totalmente el requisito de la inminencia y se anula el grado de certeza del ataque, que resulta siendo del todo aleatorio. El ataque a Soleimani se inserta en este marco jurídico.

Como se dijo antes, poco después del ataque a Soleimani el Departamento de Defensa de EE. UU. comunicaba que esta «acción defensiva» estaba justificada debido al «activo desarrollo de planes de ataque» contra objetivos estadounidenses por el general iraní, y por el hecho que él «aprobó los ataques a la embajada de los EE. UU. en Bagdad». Por lo tanto, el asesinato de Soleimani «tenía como objetivo disuadir futuros planes de ataque iraníes» («at deterring future iranian attacks») (U.S. 
Department of Defense, 2 de enero de 2020). Pocas horas después del asesinato de Soleimani, el mismo presidente Trump declaraba que «Soleimani was plotting imminent and sinister attacks on American diplomats and military personnel but we caught him in the act and terminated him» (Hosenball, 3 de enero de 2020; resaltado ańadido); así como el secretario de Estado, Michael Pompeo: "[Soleimani] was actively plotting in the region to take actions - a big action, as he described it - that would have put dozens if not hundreds of American lives at risk. We know it was imminent» (U.S. Department of State, 3 de enero de 2020; resaltado añadido).

Además, el 8 de enero, el representante permanente de los EE. UU. enviaba una nota al Consejo de Seguridad (CdS):

[d] e conformidad con el artículo 51 de la Carta de las Naciones Unidas... los Estados Unidos han adoptado determinadas medidas en ejercicio de su derecho inmanente de legítima defensa $[\ldots]$ en respuesta a una escalada de ataques armados cometidos en los últimos meses [...] a fin de impedir que la República Islámica de Irán realice o apoye nuevos ataques contra los Estados Unidos o los intereses estadounidenses, e inhibir la capacidad de las milicias apoyadas por la República Islámica de Irán y la Fuerza Quds del Cuerpo de Guardianes de la Revolución Islámica para llevar a cabo ataques». (Documento NU S/2020/20, 9 de enero de 2020; resaltado ańadido)

Estas declaraciones merecen un poco de atención.

En primer lugar, después de la acción armada contra Soleimani, EE. UU. esperó cinco días para transmitir una nota al CdS para informarlo de la situación. Esta espera demuestra, cuando menos, una escasa consideración con respecto al CdS, si se tiene en cuenta que, según lo requerido por el artículo 51 de la Carta, un Estado que usa la fuerza armada en legítima defensa tiene que comunicar «inmediatamente al Consejo de Seguridad» las medidas tomadas.

Dicho esto, la nota del representante de EE. UU. en el CdS pone atención a «a una escalada de ataques armados cometidos en los últimos meses» para justificar el asesinato de Soleimani. En particular, esta serie de ataques consistiría en un ataque por un misil tierra-aire iraní a un dron de vigilancia en el estrecho de Hormuz el 19 de junio de 2019; en la amenaza llevada a cabo por un dron iraní, el 18 de julio de 2019, a un buque de asalto anfibio en navegación en el estrecho de Hormuz mientras estaba participando en la Operación Resolución Inherente; y en ataques contra buques comerciales en el Golfo de Omán amenazando la libertad de navegación.

Por lo que respecta al primer «ataque» denunciado en la nota, cabe tener en cuenta que no todas las acciones hostiles con uso de la fuerza constituyen un ataque armado: como lo ha destacado la CIJ, «it will be necessary to distinguish the most grave forms of the use of force (those constituting an armed attack) from other less grave forms» 
(ICJ, 1986, párrafo 191); por lo tanto, parece muy dudoso que el derribo de un dron pueda constituir una de las formas más graves de ataque armado, de las que pueda surgir un derecho de legítima defensa. En cuanto a la amenaza del dron iraní, como lo definió la nota estadounidense no se trata de un ataque armado sino de una [...] amenaza. Y también pueden surgir dudas en relación con la responsabilidad de Irán por los ataques contra buques comerciales que, según la nota, habrían puesto en peligro la paz y la seguridad: de un lado, no parece que ningún país involucrado en estos ataques haya pedido socorro a EE. UU. —así que no se puede invocar la legítima defensa colectiva; de otro lado, la determinación de un peligro a la paz y a la seguridad, así como el mantenimiento de la paz, es tarea del CdS. Además, parece también dudoso que el hecho de poner obstáculos a la navegación internacional pueda constituir un ataque armado que justifique una reacción en legítima defensa por parte de EE. UU.

La nota estadounidense se refiere también a una serie de «ataques de fuego indirectos» realizados por milicias iraquíes apoyadas por la fuerza Al-Quds de Soleimani, como el ataque de 27 de diciembre de 2019 contra una base de EE. UU. en Iraq (causando la muerte de un contratista) y el ataque contra la embajada estadounidense en Bagdad (causando daños al edificio), el siguiente 31 de diciembre. De hecho, la nota no explica el punto central de este aspecto, es decir cómo Irán proporcionó su apoyo a estas milicias iraquíes. Solo ataques armados atribuibles directamente a Irán podrían haber eventualmente accionado el derecho de legítima defensa contra ese país, así que el simple apoyo (por encima de todo, inexplicado) no parece suficiente para determinar una imputabilidad directa a Irán. Este principio parece bien aclarado por el proyecto de la Comisión del Derecho Internacional sobre la Responsabilidad del Estado por hechos internacionalmente ilícitos de 2001, que destaca que «[s]e considerará hecho del Estado según el derecho internacional el comportamiento de una persona o de un grupo de personas si esa persona o ese grupo de personas actúa de hecho por instrucciones o bajo la dirección o el control de ese Estado al observar ese comportamiento" (art. 8) (Documento NU A/RES/56/83, 28 de enero de 2002).

En verdad, por lo que respecta a la cuestión de la imputabilidad a un Estado por hechos o comportamientos de un grupo no estatal, la jurisprudencia internacional ha formulado dos teorías. En el caso Nicaragua - Estados Unidos, la CIJ destacó la falta de pruebas para determinar un control efectivo (effective control) de los EE. UU. sobre los Contras, y observó que el simple apoyo a este grupo no constituía un ataque armado:

[...] despite the heavy subsidies and other support provided to them by the United States, there is no clear evidence of the United States having actually exercised such a degree of control in all fields as to justify treating the contras as acting on its behalf» 
(ICJ, 1986,párrafo 109); «[t] he Court does not consider that the assistance given by the United States to the contras warrants the conclusion that these forces are subject to the United States to such an extent that any acts they have committed are imputable to that State» (ICJ, 1986, párrafo 116); «[f] or this conduct to give rise to legal responsibility of the United States, it would in principle have to be proved that that State had effective control of the military or paramilitary operations in the course of which the alleged violations were committed. (ICJ, 1986, párrafo 115)

En lo que respecta a la relación entre Contras y EE. UU., la CIJ destacó que «the financing, organizing, training, supplying and equipping of the contras, the selection of its military or paramilitary targets, and the planning of the whole of its operation, is still insufficient in itself... for the purpose of attributing to the United States the acts committed by the contras in the course of their military or paramilitary operations in Nicaragua (ICJ, 1986, párrafo 115); además,

[...] the Court does not believe that the concept of «armed attack» includes not only acts by armed bands where such acts occur on a significant scale but also assistance to rebels in the form of the provision of weapons or logistical or other support. (ICJ, 1986, párrafo 195)

Pero en contra de lo establecido por la CIJ, el Tribunal Penal Internacional para la ex-Yugoslavia, en el caso Tadić destacó que para determinar la responsabilidad del Estado es suficiente un control general (overall control) del grupo armado no estatal, es decir que, para atribuir las acciones ilícitas de un grupo no estatal a un Estado, no es necesario que este Estado tenga que proporcionar al grupo instrucciones para realizar específicos actos contrarios al derecho internacional:

In order to attribute the acts of a military or paramilitary group to a State, it must be proved that the State wields overall control over the group, not only by equipping and financing the group, but also by coordinating or helping in the general planning of its military activity [...] However, it is not necessary that, in addition, the State should also issue, either to the head or to members of the group, instructions for the commission of specific acts contrary to international law». (ICTY, 1999, párrafo 131; Hathaway, Chertoff, Domínguez, Manfredi, Tzeng, 2017, pp. 539 y ss)

Pero queda el hecho de que no está claro qué tipo de apoyo prestó Irán (incluso a través de las fuerza Al-Quds) a las milicias iraquíes.

Por último, no se justifica el ataque a Soleimani por el hecho de que ataques armados de Irán eran «inminentes», en tanto el propósito de esta acción era «deter... Iran from conducting or supporting further attacks». Esto parece admitir que el objetivo era impedir posibles (pero no ciertos e inminentes) futuros ataques; lo que parece traducirse en una represalia armada, por supuesto prohibida por el derecho internacional. En cambio, las declaraciones de Trump y Pompeo destacan que el asesinato de Soleimani sirvió para bloquear ataques «inminentes». 
Resulta entonces claro cómo las declaraciones de la Administración estadounidense basan el concepto de legítima defensa no en el contexto de un ataque armado en curso, sino en futuros ataques, definidos como «inminentes». Como ya se señaló, la noción de «inminencia» se relaciona con la legítima defensa preventiva, es decir, aquel uso anticipado de la fuerza cuando existe la certeza de un ataque inminente; en otras palabras, el ataque va a suceder. Pero el sentido de «inminencia», propuesto por EE. UU. en el "caso Soleimani», parece difícilmente conciliable con esta categoría de legítima defensa ${ }^{5}$. En este caso, EE. UU. traslada la noción de inminencia (ataque cierto y concreto) a la noción de legítima defensa precautoria (pre-emptive self defence). Aquí la noción de inminencia propuesta por EE. UU., ya no se considera en su dimensión temporal, y abre el camino para atribuir a esta noción el sentido de que un Estado puede usar la fuerza armada en legítima defensa cuando él considere que un ataque es necesario, a pesar de la falta de certeza respecto del cuándo y el cómo se va a producir el ataque.

El resultado final es un solapamiento entre legítima defensa preventiva y precautoria (es decir, una anulación de la preventiva) que pasa por un aumento aún mayor de la discrecionalidad de aquel Estado que invoque la fuerza armada. Y junto a este solapamiento se aprecia también una mezcla (¿confusión?) entre las nociones de «inminencia» (imminence) y "disuasión»(deterrence), como resulta de las declaraciones anteriormente mencionadas: de un lado, el asesinato de Soleimani era necesario para prevenir algunos ataques definidos inminentes (aunque indefinidos o no especificados); y del otro, esta acción hubiera disuadido a los dirigentes iraníes de planificar otras futuras acciones hostiles contra EE. UU. La cuestión es hasta qué punto estas acciones deben ser «futuras». Por otra parte, cabe recordar que algunos funcionarios de la Administración americana expresaron escepticismo sobre el asesinato de Soleimani:

[ $\mathrm{t}$ ] hat official described the intelligence as thin and said that General Suleimani's attack was not imminent because of communications the United States had between Iran's supreme leader, Ayatollah Ali Khamenei, and General Suleimani showing that the ayatollah had not yet approved any plans by the general for an attack. The ayatollah, according to the communications, had asked General Suleimani to come to Tehran for further discussions at least a week before his death. (Schmitt, Cooper, Callimac y Haberman, 4 de enero de 2020)

\footnotetext{
5 La legalidad de la legítima defensa preventiva parece ya aceptada por las Naciones Unidas, como ha destacado el informe Un mundo más seguro: la responsabilidad que compartimos, elaborado en 2004 por un grupo de expertos designados por el Secretario General: «Sin embargo, según un principio de derecho internacional bien establecido, el Estado amenazado puede recurrir a la acción militar siempre que la amenaza de agresión sea inminente, no haya otro medio de impedirla y la acción sea proporcional" (Organización de Naciones Unidas, 2 de diciembre de 2004, párrafo 188).
} 
Al tener como base la falta total de certeza sobre el hecho mismo, el cómo y el cuándo, la acción disuasoria estadounidense parece más bien sustanciarse en una represalia armada.

\section{La reacción iraní}

El mismo 3 de enero, justo después del asesinato de Soleimani, el representante de Irán ante las Naciones Unidas transmitía al CdS una nota en la que comunicaba que «Irán se reserva todos sus derechos, de conformidad con el derecho internacional, de adoptar las medidas que sean necesarias al respecto, en particular en ejercicio de su derecho inmanente de legítima defensa» (Documento NU S/2020/13, 3 de enero de 2020).

Como reacción al asesinato de Soleimani, la noche del 8 de enero Irán lanzó una decena de misiles balísticos contra dos bases estadounidenses en Iraq. Con una nota transmitida ese mismo día al CdS, el representante iraní comunicaba que:

[...] ejerciendo el derecho inmanente de legítima defensa conforme al Artículo 51 de la Carta de las Naciones Unidas, las fuerzas armadas de la República Islámica de Irán emprendieron y concluyeron una respuesta militar mesurada y proporcionada cuyo blanco fue una base aérea estadounidense ubicada en el Iraq... La operación fue precisa, tuvo por blanco objetivos militares y no causó daños colaterales a la población civil ni a los bienes civiles de la zona... Irán... recuerda su dedicación al mantenimiento de la paz y la seguridad internacionales y subraya que no busca la escalada de las tensiones ni la guerra». (Documento NU S/2020/19, 8 de enero de 2020)

Por un lado, al declarar que no busca una guerra, esta nota confirma el hecho de que Irán no se considera en conflicto armado con los EE. UU.; pero, del otro, aunque se reclame correctamente (destacando la proporcionalidad de la acción armada y su conclusión), la referencia a la legítima defensa no parece justificada. El ataque estadounidense contra Soleimani había terminado e Irán no tenía ninguna razón para reaccionar en legítima defensa, salvo que siguiera esa interpretación particularmente permisiva de legítima defensa aplicada por los propios Estados Unidos, es decir, para disuadir futuros ataques. Pero, en este caso, Irán se encontraría en la circunstancia paradójica de compartir con el país rival la misma situación ilegítima, esto es, un uso de la fuerza más parecido a una represalia armada que a una reacción de legítima defensa. Este ataque iraní resulta ilícito por la misma razón que el ataque estadounidense también lo fue.

\section{Conclusiones}

La acción armada llevada a cabo por EE. UU. contra Soleimani se inserta en la política estadounidense que no reconoce la necesidad de un umbral de intensidad del 
uso de la fuerza para reaccionar en legítima defensa en caso de ataque armado. Para EE. UU. los dos términos — «ataque armado» y «uso de la fuerza»— son esencialmente sinónimos, como destaca su Manual de Guerra de 2015:

The United States has long taken the position that the inherent right of self-defense potentially applies against any illegal use of force. (U.S. Department of Defense, 2015, p. 47; resaltado ańadido)

Como ya se señaló, esta posición fue rechazada por la CIJ. Además, considerar justificada una acción armada en legítima defensa por el hecho de que un Estado suministra armas, financiaciones y apoyo logístico a grupos no estatales podría tener como consecuencia un aumento de los conflictos, incluyendo también las guerras contra el propio Estados Unidos! De hecho, Washington apoya a los grupos armados antigubernamentales en Siria con dinero, inteligencia, armas y entrenamiento, al Ejército Sirio Libre (que combate contra el Gobierno sirio) y a las Fuerzas Democrática Sirias (guerrilleros kurdos que luchan contra Siria y Turquía). Si queremos llevar este asunto al extremo, también EE. UU. podría ser objeto de ataques en legítima defensa por Siria y Turquía; así como Rusia podría actuar en legítima defensa colectiva para proteger al Gobierno sirio contra el propio EE. UU.

El pasado 3 de mayo el presidente venezolano Nicolás Maduro anunció que su Gobierno había parado un ataque "terrorista" contra Venezuela, acusando al Presidente estadounidense de haber apoyado el golpe de Estado. Entre las personas detenidas se encontraban dos ciudadanos estadounidenses y la operación fue organizada por un antiguo miembro de las fuerzas especiales americanas, hoy dirigente de una agencia de seguridad privada (Faiola, DeYoung y Herrero, 7 de mayo de 2020). Por supuesto, la Administración estadounidense negó ser el autor del intento de golpe de Estado o de participar de alguna manera en el mismo (Klein, 5 de mayo de 2020); aunque todos pensaron que detrás de este intento de golpe podría haber estado EE. UU. Si, según el asesor de Seguridad Nacional del presidente Trump, John Bolton, un conflicto armado contra Irán se justifica sobre la base de la legítima defensa, como respuesta a su apoyo a grupos armados, incluso en caso de ausencia de certeza de la existencia de dicha ayuda (Hathaway, 31 de mayo de 2019) jesta mera sospecha podría justificar una reacción armada en legítima defensa de Venezuela para disuadir e impedir posibles futuros ataques contra el Gobierno venezolano?

Cabe subrayar otro aspecto del «caso Soleimani». Hoy en día, en nuestro criterio, la legítima defensa preventiva es generalmente aceptada, así que «el Estado amenazado puede recurrir a la acción militar siempre que la amenaza de agresión sea inminente», como lo señala el informe de las Naciones Unidas Un mundo más seguro (documento NU A/59/565, 2 de diciembre de 2004, párrafo 188). El informe atribuye al 
término «inminente» el significado literal y natural de "próximo», desde el punto de vista temporal, y rechaza la noción «estadounidense» de legítima defensa precautoria (documento NU A/59/565, 2 de diciembre de 2004, párrafo 189 y ss.). Con el asesinato de Soleimani la «fantasía jurídica» de la Administración estadounidense ha alcanzado nuevas metas: un ataque armado no es inminente cuando sea seguro y se materialice, sino cuando EE. UU. decida que la respuesta armada es necesaria. Podría ser que ni Trump, ni Pompeo se hayan dado cuenta que inventaron una nueva categoría ilícita de legítima defensa, es decir la «legítima defensa precautoria inminente».

\section{Referencias bibliográficas}

Callamard, A. The Targeted Killing of General Soleimani: Its Lawfulness and Why It Matters. Just Security. Recuperado de www.justsecurity.org/67949/ the-targeted-killing-of-general-soleimani-its-lawfulness-and-why-it-matters

Dinstein, Y. (2016). The Conduct of Hostilities under the Law of International Armed Conflict. Cambridge: Cambridge University Press. https://doi.org/10.1017/ CBO9781316389591

Faiola, A., DeYoung, K. y A.V. Herrero. (7 de mayo de 2020). De Miami a Venezuela: así falló el plan de 'capturar' a Maduro. The Washington Post. Recuperado en www.washingtonpost.com/world/2020/05/07/de-miami-venezuela-fall-el-plan-de-capturar-maduro

Garland, C. (28 de diciembre de 2019). American Defense Contractor Killed, Troops Wounded in Rocket Attack on Base in Kirkuk. Recuperado de www.stripes.com/ news/middle-east/american-defense-contractor-killed-troops-wounded-in-rocketattack-on-base-in-kirkuk-1.612677

Gilsinan, K. (4 de enero de 2020). It Wasn't the Law that Stopped other Presidents from Killing Soleimani. Recuperado de www.theatlantic.com/politics/archive/2020/01/ why-kill-soleimani-now/604441

H.E. Prime Minister Adil Abd Al-Mahdi Receives the US Ambassador (6 de enero de 2020). Recuperado de https://pmo.iq/pme/press2020en/6-1-201201en.htm

Hathaway, O. (31 de mayo de 2019). Bolton's Stated Predicate for War with Iran Doesn't Work. Just Security. Recuperado de www.justsecurity.org/64372/ john-boltons-stated-predicate-for-war-with-iran-doesnt-work-proxies

Hathaway, O., Chertoff, E., Domínguez, L., Manfredi, Z. y Tzeng, P. (2017). Ensuring Responsibility: Common Article 1 and State Responsibility for Non-State Actors. Texas Law Journal, 95, 539-590.

Hosenball, M. (3 de enero de 2020). Trump Says Soleimani Plotted 'Imminent' Attacks, but Critics Question Just How Soon. Reuters. Recuperado de www.reuters.com/article/ us-iraq-security-blast-intelligence/trump-says-soleimani-plotted-imminent-attacksbut-critics-question-just-how-soon-idUSKBN1Z228N

International Committee of the Red Cross. (2011). International Humanitarian Law and the Challenges of Contemporary Armed Conflicts, $31^{\text {st }}$ International Conference of the Red Cross and Red Crescent. Ginebra. Recuperado de https://www.icrc.org/en/doc/assets/ 
files/red-cross-crescent-movement/31st-international-conference/31-int-conferenceihl-challenges-report-11-5-1-2-en.pdf

International Court of Justice. (27 de junio de 1986). Case concerning Military and Paramilitary Activities in and against Nicaragua, Judgment. Recuperado de https://www.icj-cij.org/ files/case-related/70/070-19860627-JUD-01-00-EN.pdf

International Court of Justice. (2011). Case Concerning the Aerial Incident of 3 July 1988: Islamic Republic of Iran v. United States of America. V. 2: Pleadings, Oral Arguments, Documents.

International Court of Justice. (6 de noviembre de 2003). Case Concerning Oil Platforms (Islamic Republic of Iran v. United States of America), Judgment. Recuperado de https:// www.icj-cij.org/files/case-related/90/090-20031106-JUD-01-00-EN.pdf

International Law Association. (2010). Final Report on the Meaning of Armed Conflict in International Law. Recuperado de http://www.rulac.org/assets/downloads/ILA_ report_armed_conflict_2010.pdf

International Tribunal Former Yugoslavia. (2 de octubre de 1995). Prosecutor v. Tadić, Decision on the Defence Motion for Interlocutory Appeal on Jurisdiction.

Kelly, J.B. (1965). Assassination in War Time. Military Law Review, 30, 101-111.

Klein, B. (5 de mayo de 2020). Trump dice que el incidente en Venezuela «no tiene nada que ver con nuestro gobierno». CNN. Recuperado de https://cnnespanol.cnn. com/2020/05/05/trump-dice-que-el-incidente-en-venezuela-no-tiene-nada-que-vercon-nuestro-gobierno

Loveluck, L., Taylor, A. y Brice-Saddler, M. (3 de enero de 2020). Trump Says Iranian Military Leader Was Killed by Drone Strike 'to Stop a War,' Warns Iran not to Retaliate. The Washington Post. Recuperado de www.washingtonpost.com/world/iran-strike-liveupdates/2020/01/03/3779f55c-2e33-11ea-bcb3-ac6482c4a92f_story.html

Organización de Naciones Unidas. (2 de diciembre de 2004). Un mundo más seguro: la responsabilidad que compartimos. Documento NU A/59/565. Recuperado de https:// undocs.org/pdf?symbol=es/A/59/565

Pictet, J. (1952). Commentary I Geneva Convention for the Amelioration of the Condition of the Wounded and Sick in Armed Forces of the Field. Ginebra: Comite Internacional de la Cruz Rojo. Recuperado de https://www.loc.gov/rr/frd/Military_Law/pdf/ GC_1949-I.pdf

Schmitt, E., Cooper, H., Callimac, R. y Haberman, M. (4 de enero de 2020). As Tensions with Iran Escalated, Trump Opted for Most Extreme Measure. The New York Times. Recuperado de www.nytimes.com/2020/01/04/us/politics/trump-suleimani. html?campaign_id=60\&instance_id=0\&segment_id=20060\&user_id=285e0687bd 79f5610c20b52d37875ff8\&regi_id=47276260

Smith, C. (5 de enero de 2020). United States Killed Iraqi Military Official and Iraqi Military Personnel in the Two Recent Attacks. Just Security. Recuperado de www.justsecurity. org/67917/united-states-killed-iraqi-military-official-and-iraqi-military-personnelin-the-two-recent-attacks 
Smith, C. y Singer-Emery, J. (2019). Servants of Two Masters: The Risks Inherent in Iraq's Hashd Al-Sha'abi Legislation. New York University Journal International Law and Politics, 52(1), 167-229. Recuperado de https://papers.ssrn.com/sol3/papers. cfm?abstract_id=3511792

Sofaer, A.D. (1989). The Sixth Annual Waldemar A. Solf Lecture in International Law. Terrorism, the Law, and the National Defense. Military Law Review, 126, 89-124. Recuperado de https://www.loc.gov/law/mlr/Military_Law_Review/277079 1.pdf

Solis, G. (2007). Targeted Killing and the Law of Armed Conflict. Naval War College Review, 60(2), 127-146. Recuperado de https://digital-commons.usnwc.edu/nwc-review/ vol60/iss $2 / 9$

Taft, W. (2004). Self-Defense and the Oil Platforms Decision. Yale Journal of International Law, 29(2), 295-306. Recuperado de https://digitalcommons.law.yale.edu/yjil/ vol $29 /$ iss $2 / 3$

The National Security Council Holds a Meeting Chaired by His Excellency the Prime Minister and Commander-in-Chief of the Armed Forces Adil Abd Al-Mahdi (3 de enero de 2019). Recuperado de https://pmo.iq/pme/press2020en/3-1-201201en.htm

U.S. Department of Defense. (2015). Law of War Manual (Updated December 2016). Washington D.C. Recuperado de https://dod.defense.gov/Portals/1/Documents/ pubs/DoD\%20Law\%20of\%20War\%20Manual\%20-\%20June\%202015\%20 Updated\%20Dec\%202016.pdf?ver=2016-12-13-172036-190

U.S. Department of Defense. (29 de diciembre de 2019). Statement from Assistant to the Secretary of Defense Jonathan Hoffman. Recuperado de www.defense.gov/Newsroom/Releases/Release/Article/2047960/ statement-from-assistant-to-the-secretary-of-defense-jonathan-hoffman

U.S. Department of Defense. (2 de enero de 2020). Statement by the Department of Defense. Recuperado de www.defense.gov/Newsroom/Releases/Release/Article/2049534/ statement-by-the-department-of-defense

U.S. Department of State. (3 de enero de 2020). Secretary Michael R. Pompeo with John Berman of CNN New Day - Interview. Recuperado de www.state.gov/ secretary-michael-r-pompeo-with-john-berman-of-cnn-new-day

U.S. The White House. (2002). National Security Strategy of the United States of America, 2002. Recuperado de https://2009-2017.state.gov/documents/organization/63562.pdf

Villalaz, J.Y. (21 de enero de 2020). Soleimani: terrorismo, drones y derecho internacional. Globalización. Centro de investigación sobre Globalización. Recuperado de www.globalizacion.ca/soleimani-terrorismo-drones-y-derecho-internacional

Recibido: 11 de mayo de 2020

Aprobado: 21 de agosto de 2020 